\title{
Hyperbaric Oxygen Therapy for 'Happy' Hypoxia in COVID-19 Patient, Why is it Better?
}

\author{
Sofia Wardhani ${ }^{1,2 *}$, M Guritno Suryokusumo ${ }^{3,4}$, Naura Rahma Andhika Putri ${ }^{5}$ \\ ${ }^{1}$ Doctoral Program, Faculty of Medicine, Universitas Airlangga, Indonesia \\ ${ }^{2}$ Lecturer, Faculty of Health, UPN 'Veteran' Jakarta \\ ${ }^{3}$ Subaquatic and Hyperbaric Medicine Academie Nationale de Medecine, Paris, France \\ ${ }^{4}$ Chairman of UPN 'Veteran' Jakarta Health Research Ethic Committee \\ ${ }^{5}$ Undergraduated Program, Faculty of Medicine, Dentistry and Health Sciences, University of Melbourne, \\ Australia \\ *Corresponding author. Email : sofia_wardhani@upnvj.ac.id
}

\begin{abstract}
Background: Lately, there has been many discussions regarding happy / silent hypoxia, whereas the patient does not sense or show symptoms of breathlessness yet the patient has been experiencing hypoxia due to Covid-19 infection. This has caused death to many Covid-19 patients without comorbidities. The most common therapy that is given to COVID-19 patients who experience worsened breathing is to use a mechanical ventilator. However, this therapy has not provided satisfactory result while Hyperbaric Oxygen Therapy (HBOT) has been used to treat various disease that lead to hypoxia, including $\mathrm{CO}$ poisoning and chronic anemia. Objective: to explore the possible pathophysiology underlying happy/silent hypoxia in COVID19 patients, its consequences to oxygen debt and how HBOT is a better treatment compared to mechanical ventilation. Method: This study uses the literature review method using electronic database in the form Pubmed, SCOPUS, Science Direct, Open Access Library (OALIB), Research gate and Google Scholar with restrictions on the keywords of HBOT, Happy hypoxia and COVID-19. Result: Reports from multiple hyperbaric centres in China and the USA showed that patients receiving HBOT treatment as adjuvant therapy can prevent them receiving mechanical ventilation and help overcome the respiratory failure. The proposed mechanism underlying this effect is due to its noninvasive, anti-inflammatory properties and the ability to increase alveolar- arterial oxygen partial pressure $(\mathrm{PA}-\mathrm{a}) \mathrm{O}_{2}$. Although this therapy has only been given to a small number of COVID-19 patients, the results obtained are that most of these patients experience rapid and permanent improvement without any serious side effect that can worsen the condition. Conclusion: Reports from various Hyperbaric Centres in China and the USA show promising results in using HBOT to overcome the respiratory failure in COVID-19 due to its various properties and with ongoing RCTs, it is hoped that it will give satisfactory results so that it can help overcome hypoxia in COVID-19 patients.
\end{abstract}

Keywords: HBOT, COVID-19, Happy/Silent hypoxia, Oxygen debt

\section{INTRODUCTION}

First identified in Wuhan, China on 2019, SARS CoV-2 is the newest member of the enveloped RNA betacoronavirus family. It receives the name of Severe Acute Respiratory Syndrome coronavirus 2 because of its similarities with SARS CoV and Middle East Respiratory Syndrome (MERS)[1] . SARS CoV-2 as the cause of
Covid-19 is the second form of SARS which had caused a global pandemic spread approximately to 29 countries in 2002 . This illness has a quite wide severity spectrum and is classified to mild $(81 \%)$, severe $(14 \%)$ or critical $(5 \%)$ [2] where breathing failure is the main cause of death due to SARS virus[3]. The failure in the mechanism of the host's tissue due to the 
virus can be an immediate process to target cells or by a prolonged inflammatory response in the body, which is now known as "cytokine storm"[4]. Unfortunately, lately some cases have been found where patients do not mention any symptoms resembling those commonly found in pneumonia patients yet rapidly fell into a critical condition due to breathing failure. Medical experts defined this with silent or happy hypoxia, and has been reported through a number of electronic media in Indonesia.

This condition should be given attention because patients that have suffered from chronic hypoxia would eventually lead to an oxygen debt. Standard therapy for COVID 19 patients are often difficult to be delivered properly as lung inflammation that is caused by the virus is limiting oxygen uptake by haemoglobin. This is where HBOT plays an adjuvant therapy in patients with silent hypoxia because it can rapidly increase dissolved oxygen levels in plasma and "pay" the oxygen debt. this paper focuses on the mechanism of happy hypoxia and why HBOT is a more suitable tool for therapy than mechanical ventilator. In addition, the authors also informed about which countries that already used HBOT as an adjuvant therapy for COVID-19 patient and the obtained result.

\section{METHOD}

This study uses the literature review method using electronic database in the form of Pubmed, SCOPUS, Science Direct, Open Access Library (OALIB), Research gate and Google Scholar with restrictions on the keywords of HBOT, Happy hypoxia and COVID-19. We started the research from the first paper in China that reports the use of HBOT as an adjuvant therapy for 35

COVID-19 patients with encouranging result and the recent concern about happy hypoxia causing death to COVID-19 patients without any prior respiratory problem or comorbidites. However we only found four papers that report about the use of HBOT for COVID-19 patient in the form of case report and two of the paper were written in Chinese language so we used other review articles that contain these two reports. Since there is no result yet from RCTs about HBOT and COVID-19, we explore the possibility of pathophysiology mechanism for infection and happy hypoxia that also occur in other disease to point out the benefits of HBOT.

\section{RESULT \\ Acute Respiratory Distress Syndrome (ARDS)}

In the event of a tissue infection, the body will activate an innate immune response, which consists mainly of neutrophils and monocytes. There cells release various toxic agents in the form of chemokine (CCL-2, CCL3, and CCL5 ) and cytokine (IL- $1 \alpha$, IL-1 $\beta$, IL-10, TNF- $\alpha$ and, IL-6 ) which not only kill pathogens but also damages the surrounding tissue, causing necrosis[5][6][7]. Necrosis forms debris from dead cells that can activate a secondary inflammation response by further recruitment of neutrophils and macrophage among other immune systems to the inflamed site. Macrophage will further release more cytokine so that there will be a vicious circle that eventually destroys normal surrounding tissue (cytokine storm)[8]. Upregulation of cytokine and chemokine responses causes endothelial cell apoptosis which can lead of pulmonary microvascular and alveolar epithelial cell barriers damage that will lead to leakage of blood vessels and alveolar edema[9][10][11][12] . Host responses to uncontrolled infection in lung tissue causes Acute Respiratory Distress Syndrome (ARDS) and hypoxia as symptoms commonly found in patients with sepsis due to viral, bacterial, fungal infection or following a trauma or injury[13][14][15]. In COVID-19 patients, high cytokine levels correlate positively with disease severity and mortality[4] and cytokine storm can be observed in some patient which generally lead to ventilator use and eventually death. 
If this cytokine storm is released from the lung tissue and enters the circulation it can cause damage to other organ such as the brain, kidneys and heart[16] and can end up in multiple organ failure or multiple organ dysfunction syndrome which will later be referred as MOF and MODS succesively throughout this paper[5]. Thus especially in COVID-19 patients, secondary organ damage may not be a direct result of infection with the SARS CoV-2 virus but also the result of an uncontrolled inflammatory response in the body that can lead to shock, MOF and eventually death. Based on the Berlin definition, Acute respiratory distress syndrome is a condition in which the arterial oxygen concentration is very low i.e. hypoxia, with a ratio of arterial oxygen partial pressure to inspired ox ygen fraction $(\mathrm{PaO} 2 / \mathrm{FiO} 2$ ratio) less than 300 accompanied with bilateral thickening of lung tissue[17]. ARDS is an acute, diffuse, inflammatory lung injury with increased pulmonary vascular permeability, increased lung weight and loss of aerated lung tissue. Determination of diagnosis usually one week from the appearance of clinical signs or any new or worsening respiratory symptoms. Typical clinical signs of ARDS are hypoxemia and bilateral radiographic opacities (severe ARDS if the opacities cover 3 or 4 quadrants), increased physiological dead space and decreased pulmonary compliance[17].

Respiratory failure due to ARDS and MOF is the main cause of COVID19 death which is supported by limited postmortem data showing alveolar edema, proinflammatory concentrations, fibrin deposition in pneumocytes and indications of early phase of ARDS[18].

\section{HYPOXEMIA, HYPOXIA,}

\section{HYPERCAPNIA, DYSPNEA}

Oxygen is an essential molecule for aerobic organism to make cell energy (ATP) to maintain cellular viability through mitochondrial electron transport chain (METC) or known as mitochondrial respiratoy chain. Normoxia condition maintain ATP/ADP ratio high and constant so if there is a decreased in oxygen supply (hypoxia) up to a certain level then the cell cannot maintain its function and causing decreased cell viability[19][20].

Oxygen are obtain via respiration with medulla oblongata respiratory center and pons regions of the brainstem controlled respiratory drive, to match respiration and body metabolic need[21][22]. The respiratory drive is getting their feedback from central and perifer chemoreceptor. Central respiratory also influenced by higher brain cortex, hypothalamic integrative nociception, feedback from mechanostrectch receptors in muscle and lung and also metabolic rate. The output of respiratory centre are rhythm (respiratory rate) and pattern generating (depth of breathing effort) signals and both can be independent[23]. Before going deep into the discussion, we need to distinguish terminology between hypoxemia, hypoxia, hypercapnia dan dyspnea.

\section{Hypoxemia}

Although in some publication it been used interchangeable with hypoxia, it still have different meaning. Hypoxemia is a condition or state where there is a low arterial oxygen supply or decreased partial pressure of oxygen in blood. The normal response to hypoxemia is an increase in minute ventilation especially with an increase in tidal volume and respiratory rate. In healty subject, mild hypoxemia ( $\mathrm{PaO} 260$ - $65 \mathrm{mmHg}$ ) can caused minimal change in respiratory drive. This condition usually occur in high altitude or undergo hypoxic chambers[22][24]. Hypoxemia itself has a limited role in terms of the sensation of breath experienced by patients with cardiopulmonary disease as opposed to hypercapnia which can cause dyspnea per se[21][25][26]. Increased respiratory rate (tachypnea) and tidal volume (hyperpnea) instead of dyspnea are important clinical signs of impending hypoxemic respiratory failure[22][27]. 


\section{Hypoxia}

Hypoxia is reduced level of tissue oxygenation so that the cell cannot do its normal function. Ischemia different from hypoxia because in ischemia there is also reduction of blood flow that is not only disturb oxygen supply but also nutrition. This reduction also caused accumulation of metabolic product such as $\mathrm{CO}_{2}$, lactic acid and ammonia[28][29][30]. Based on time of onset, hypoxia can be devided into acute (second to minute), intermediate and chronic (hours to days), and also can be devided based on oxygen concentration (moderate $\left(5-8 \% \mathrm{O}_{2}\right)$ and anoxic $\left(<1 \% \mathrm{O}_{2}\right)$ $\begin{array}{lll}\text { (normoksia } 21 \% & \mathrm{O} 2)[31][32][33] \text {. }\end{array}$ Hyperventilation, increased cardiac output, systemic arterial vasodilatation, vasoconstriction, relaxation of ductus arteriosis, activation of glucose uptake are some of the acute response to hypoxia. Meanwhile, chronic response to hypoxia are increased glucose metabolism and transport in most of the tissue, eritropoiesis, angiogenesis, and neovascularization. Eventually this lead to hypertrophy and remodelling arterial pulmoner wall, myocardium, carotid body and vasodilator production.

\section{Hypercapnia}

Hypercapnia is the presence of excessive amounts or partial pressure of carbonmonoxide in the blood. The changes of blood $\mathrm{CO}_{2}$ partial pressure $\left(\mathrm{PaCO}_{2}\right)$ are important chemical component causing $\mathrm{pH}$ shift at the level of peripheral and central chemoreceptors[21][22][34]. In steady state, Arterial $\mathrm{PaCO} 2$ can be measure with this equation.

$$
\mathrm{PaCO} 2=\underset{\mathrm{Ve} \cdot(1-\mathrm{Vd} / \mathrm{Vt})}{\mathrm{K} \cdot \mathrm{VCO} 2}
$$

$\mathrm{K}$ : Constant (863 mmHg), VCO2 : rate of $\mathrm{CO} 2$ production, Ve: minute ventilation. Vd: Dead space, Vt: Tidal

$$
\text { Volume[23] }
$$

According to the equation above, hypercapnia can result from increased $\mathrm{Vd}$, Hypoventilation or increased $\mathrm{VCO}_{2}$ and the normal response to that is increased respiratory drive and minute volume ventilation[35].

$\mathrm{PaCO} 2$ is also one of the fundamental regulator in cerebral blood flow. Decreased of $\mathrm{PaCO} 2$ caused by hyperventilation can cause arterial vasoconstriction that will decreased cerebral blood flow and increased intracranial pressure. Conversely, an increase in $\mathrm{PaCO} 2$ causes an increase in intracranial pressure ultimately leading to a deteriorating level of consciousness, altered brainstem, postural and motor responses[36][37].

\section{Dyspnea}

Dyspnea is a sensation of discomfort, difficulty or heaviness during breathing that occurs when the need for ventilation exceeds the patient's ability and is different from tacypnea (rapid breathing) or hyperpnea (increased ventilation). Many patients who experience dyspnea are not hypoxemic, while those who are, generally experience slight symptom improvement when hypoxemia is corrected by addition of oxygen therapy. Generally dyspnea often occurs when arterial $\mathrm{PaO} 2$ falls below 40 $\mathrm{mmHg}[21]$ or when there is a buildup of $\mathrm{CO}_{2}$ in the tissue.

\section{HAPPY/SILENT HYPOXIA}

The prominent feature from COVID19 patients is hypoxia due to respiratory failure. However recently there are some report that patient COVID-19 were admitted to the hospital with low oxygen saturation but without prior symptom of dyspnea. Guan reported that dyspnea only found in $18,7 \%$ from 1099 pasien COVID19 who were hospitalized, despite rasio $\mathrm{PaO} 2 / \mathrm{FiO} 2$, abnormal CT scans (86\%) and use of supplemental oxygen (41\%)[38]. The same result was found by Dr Richard Levitan from Bellevue Hospital New York who observed the blood oxygen levels of COVID-19 patients where they experienced arterial hypoxemia but did not show equivalent respiratory distress and did not even complain of dyspnea[39][40][41][42]. Even a decrease 
in oxygen saturation from normal (94$100 \%$ ) to $50 \%$ as in three cases presented by Tobin $\mathrm{MJ}$ [43] with $\mathrm{PaO}_{2}$ ranging between 36 and $45 \mathrm{mmHg}$ and $\mathrm{PaCO}_{2}$ between 34 and $41 \mathrm{mmHg}$ without an increase in alveolar ventilation and complaints of dyspnea.

The discrepancy between the severity of hypoxemia and mild respiratory distress is called 'silent/happy hypoxia' which is in contrast to the experience of doctors take care of critical patients with respiratory failure[41]. Happy hypoxia in COVID-19 patients can even occur in the early stages of pneumonia symptoms[44]. Happy or silent hypoxemia can also occur in patients with atelectasis, intrapulmonary shunt due to arterio-venous malformation or right to left intracardiac shunt. Several mechanisms are thought to contribute to the development of arterial hypoxemia in COVID-19 patients, including idiosyncratic effects on the respiratory control system and pulmonary thrombi vasculature.

Cell receptor of SARS-CoV-2, which is called ACE2 (angiotensin - converting enzyme 2) is present in the carotid body, a chemoreceptor that is sense oxygen[45] and nasal mucosa. Two thirds of COVID - 19 patient suffer from anosmia - hyposmia[46] and there is a pathway to the brain through the olfactory bulb as access for coronavirus to entry the brain[47]. However, it remains to be further studied wheter this access exists and contributes to the relationship of anosmia - hyposmia and dyspnea[46] and whether ACE2 receptors have a role in reducing the dyspnea response to COVID19 should further studied.

There are several mechanisms that can cause hypoxemia in COVID 19 patients, including intrapulmonary shunting, loss of lung perfusion regulation, intravascular microtrombi, impaired diffusion capacity, preservation of lung mechanics and ending with Rapid deterioration[23]. This paper will only focus on the mechanism in which HBOT has more role than mechanical ventilation.
Adequate gas exchange is determined primarily by the balance between pulmonary ventilation and capillary blood flow which called ventilation/perfusion (V/Q) matching[48]. COVID-19 patient experienced hypoxemia in the early stages of the disease as a result of V/Q mismatch. In this condition, there is a result of the lung area experiencing an increased in the V/Q ratio and other areas experiencing a decrease in the V/Q ratio. In addition, due to inflammation in some part of the alveolar, there is a buildup of inflammatory exudate which result in the blood flowing to the area not getting perfused (dead space) which is marked by an increase in the gradient of alveolar- arterial oygen partial pressure (PA-a) $\mathrm{O}_{2}$. Exudate will cause pulmonary edema which is characterized by an appearance of groundglass opacities and consolidation on chest X-rays, reduced surfactant and superimposed pressure, collapse alveolar and this result in an area that is not properly ventilated which is called intrapulmonary shunting[27]. As the disease progress, there is an increase in tidal volume resulting in an increase in negative inspiratory intrathoracic pressure coupled with an increase in lung permeability due to inflammation resulting in progressive edema, alveolar flooding and patient self inflicted lung injury. This overall results in an increase in the shunted lung area which is difficult to correct even with an increase in $\mathrm{FiO} 2$.

The presence of continuous pulmonary blood flow to the nonaerated area also appears to be a result of failure of the hypoxic pulmonary vasoconstriction mechanism, a response to alveolar hypoxia in the form of constricting arteries[49][50]. However the mechanism is still not known with certainty whether the result of the inflammatory process is that it releases endogenous vasodilators, namely prostaglandins, bradykinin and cytokines or others[51][52]. Another thing that is thought to be involved but still requires further research is the presence of vasoplegia due to shear stress at the 
interfaces between lung structures, dysregulation Renin Angiotensin System (RAS) since the Angiotensin Converting Enzyme 2 (ACE2) is the receptor used by SARS-CoV-2 to enter the body [53], [54], [55]. A recent report by Liu et al shows that viral load and lung injury in COVID-19 are directly proportional to serum AngII[56].

Endothelial injury is now a central hallmark of pathogenesis and this virus can directly infect capillary pulmonary endothelial cell expressing ACE2 [55][57]. As a result of acute inflammation and endothelial injury, an imbalance between procoagulant and fibrinolytic activity can occur, forming an intravascular microthrombi[58][59][60]. Many COVID-19 patients show elevated D-dimers as a marker of clot formation and this is used to predict in hospital mortality with DIC occurring in 71\% of COVID-19 patients with dismal prognosis, versus only $0,6 \%$ of survivor[61][62][63][64]. The hypercoagulation condition causes deterioration in V/Q mismatch and lung tissue damage as shown in autopsy report[64][65][66].

Viral infection into alveolar type II cells followed by an immune response can cause damage to the infected cells[55]. As a result, there is a condition where epithelial cells are lost and procoagulant state that a hyaline membrane is formed which is a denuded basement membrane that be covered with debris (fibrin, dead cells, complement activation)t[55][67]. As a result of incremental exercise and the absence of vasoconstriction, hyperdynamic pulmonary circulation occurs which cannot allow sufficient time for the red blood cells to equilibrate their oxygen uptake. This diffusion limitation resulting in an increase in $\mathrm{P}(\mathrm{A}-\mathrm{a}) \mathrm{O} 2$ gradient and Exercise-Induced Arterial Hypoxemia (EIAH)[68]. The prevalence impaired diffusing capacity is related to disease severity, namely $30.4 \%$ in mild illness, $42.4 \%$ in pneumonia and $84.2 \%$ in severe pneumonia[69].

The sigmoid image of the oxyhemoglobin dissociation curve appears to shift to the left due to increased respiratory alkalosis (decreased $\mathrm{PaCO} 2$ ) due to hypoxemia driven tachypnea and hyperpnea. During the hypocapnic period, the affinity of hemoglobin with oxygen increases and this causes oxygen saturation to increase to a certain $\mathrm{PaO} 2$ limit which explains why $\mathrm{SpO} 2$ is still normal even when $\mathrm{PaO} 2$ is low[51][70][71]. This finding can also be seen in hypoxaemia at altitude where hypocapnia significantly shifts the oxygen haemoglobin dissociation curve and improves blood oxygen saturation[23]. The alveolar gas equation also predicts that hyperventilation and a decrease in the partial pressure of $\mathrm{CO}_{2}$ in the alveolar cause an increase in alveolar oxygen partial pressure and ultimately lead to an increase in $\mathrm{SpO}_{2}$ [34]. Thus when $\mathrm{CO}_{2}$ begin to accumulate in the tissue and oxygen saturation drops at a critical point, which increases the risk of COVID-19 patients falling into respiratory failure and entering the Intensive Care Unit (ICU)[72][73]. In conclusion, $\mathrm{SpO} 2$ should be interpreted at the time of hyperventilation (Tacypnea, low $\mathrm{PaCO} 2$ ) and if necessary, $\mathrm{PaO} 2$ examination by arterial puncture.

In some COVID-19 patients, the development of gas exchange abnormalities occurs before an increase in mechanical loads[74]. At the onset of infection, there is no increase in airway resistance and it can be assumed that no dead space ventilation occurs either anatomically or physiologically. And because pulmonary compliance is still normal in many patients who previously did not have lung disease, breathing effort remains rather low as shown by Gattinoni et al. in a cohort of 16 critically ill Patients. Initially they had relatively normal values for respiratory system compliance $(50.2 \pm 14.3 \mathrm{ml} / \mathrm{cmH} 2 \mathrm{O})$ went hand in hand with a dramatically increased shunt fraction of $0.50 \pm 0.11[75]$. This sizeable difference is rarely found in disorders that can lead to acute lung injury and ARDS[75][76]. Relatively high compliance indicates a wellpreserved lung gas volume and explains in part the absence of dyspnea 
early in the course of illness[49][62][75]. In contrast, Ziehr et al. instead he obtained low compliance and symptoms consistent with ARDS according to the Berlin definition in his patient[41][69].

Couzin-Frankel J[40] links silent hypoxemia with thrombin development in the pulmonary vasculature due to an increase in thrombogenesis in COVID-19 patients COVID 19 patients[77]. Thrombi in the pulmonary vasculature can cause severe hypoxemia and dyspnea[78]. In addition, the release of histamine and stimulation of juxtacapillary receptors in the pulmonary vasculature can cause dyspnea. However, until now there is no biological mechanism that can explain whether thrombi in the pulmonary vasculature can cause blunting dyspnea, resulting in silent hypoxemia[43]. COVID19 patients with the highest disease severity and lowest respiratory system compliance ultimately have to put on mechanical ventilation and dyspnea accelerate the use of a ventilator[74].

A more comprehensive understanding of pathophysiology of respiratory drive and hypoxemia in COVID-19 patients is needed so that we can read clinical signs and determine appropriate management[22].

\section{OXYGEN DEBT}

Body tissues require $5-6 \mathrm{~mL}$ of oxygen per $100 \mathrm{~mL}$ of blood flow, with greater oxygen demand for the brain, heart and retina. Inadequate oxygenation for days in a patient can give rise to 'oxygen debt' as additional insult. However, oxygen debt is not identical with acute hypoxia. Oxygen debt is the minimum requirement for tissue oxygen consumption minus the availability of oxygen supply in liters of undersupplied oxygen per meter queared of body surface area $(\mathrm{L} / \mathrm{m} 2)$. Survival is not possible if oxygen debt exceeds $33 \mathrm{~L} / \mathrm{m}^{2}$ (Van Meter in UHMS 2020)[18]. Meanwhile, multiorgan failure can occur with oxygen debt of $22 \mathrm{~L} / \mathrm{m}^{2}$ and can survive without residual organ dysfunction or injury in debt of not more than $9 \mathrm{~L} / \mathrm{m}^{2}[18][79]$. Generally, we can observe oxygen debt in patients with severe chronic anemia, $\mathrm{CO}$ poisoning and even in sepsis. In anemic patients, critical oxygen debt occurs when the haemoglobin level drops to $6 \mathrm{mg} / \mathrm{dL}$. Oxygen debt can initiate disease exacerbation in sepsis and ARDS and if not resolved can cause major multiorgan failure[18]. In fact, Rixen and Siegel[80] stated that oxygen debt is more important in determining the severity of hemorrhagic shock compared to blood loss, volume replacement, blood pressure or heart rate.

The principle of treating patients with sepsis as in COVID-19 is to intervene as early as possible such as controlling the source of infection or injury[81][82], the use of antibiotics[83] or reducing the inflammatory response[84]. Until now, no appropriate therapeutical drug and vaccines have been found to reduce the impact of lethality and reduce the curve of COVID-19 infection therefore supportive therapy is the only option for clinicians in patient management. One of the supportive therapies according to the standard of sepsis patients is mechanical ventilation as a last resort in saving COVID-19 patients[44]. COVID-19 patients with comorbids generally experience worsening faster than those without comorbids to the point where they need a mechanical ventilator to save their lives. However, some ventilator dependent patients died in a $50-80 \%$ and the use of extracorporeal membrane oxygenation (ECMO) did not appear to benefit the group of patients with very severe symptoms[18]. That's why clinicians are trying to find effective therapies, especially to prevent COVID-19 patients from falling in a condition that requires a ventilator. Related to this, hyperbaric oxygen therapy (HBOT) can be an adjuvant therapy option to improve the outcome that can be done as soon as a decrease in arterial oxygen concentration is detected. 


\section{HBOT}

Hyperbaric Oxygen Therapy or HBOT is a therapy of $100 \%$ oxygen delivery in high pressure chamber (clinical dose of 2,4 ATA) and has been used in diving and clinical disease with a high level of safety. Some indications for the clinical use of HBOT are as the main therapy for carbon monoxide gas poisoning and gas embolism, diabetic ulcer[85][86], improve kidney function after infection[87] and reduce kidney damage in diabetic patients[88], radiation injury[89], ischemic reperfusion injury[90], improved wound healing[91], diminish UV-induced skin damage[92], extreme form acute respiratory distress syndrome[93] and reverse lethal oxygen debt in cardiac arrest[79]. HBOT was also usedduring the Spanish flu pandemic (1918) by Dr Orval Cunningham of Kansas City USA on a patient whose condition was rapidly deteriorating and cyanotic[94]. Oxygen from air binds to haemoglobin in red blood cells after diffusing through the alveolar walls of the pulmonary interstitium, capillary walls, blood plasma, red blood cell membranes, cytoplasm of red blood cells to haemoglobin. COVID-19

patients with pneumonia experience a build-up of inflammatory exudate and inflammation of the interstitium resulting in diffusion barriers in the alveoli so that oxygen uptake also decreases. In this case, it takes effort to increase the oxygen pressure of the alveoli in order to penetrate the barrier. However, standard therapy that has been used so far cannot penetrate the diffusion inhibitors in the lungs because it is limited by environmental pressure so that it cannot cope with accumulated oxygen debt and intense pulmonary and systemic inflammation.

High ambient pressure in HBOT has an impact on the respiratory gas according to Henry's Law which states that the concentration of gas in a liquid is proportional to the pressure of the gas above it[79]. This law underlie gas exchange in the lungs and is used to overcome the solubility and diffusion barrier problems of oxygen in patients with pneumonia. Increased oxygen solubility in the alveolar can penetrate the pulmonary membrane inflammatory barrier, increase the rate of oxygen diffusion, increase the oxygen diffusion distance, increase the solubility of oxygen in plasma, increase haemoglobin saturation in red blood cells and deliver oxygen to the microcirculation and tissues. Increased solubility of oxygen in plasma due to HBOT also causes oxygen to dissolve in tissues that have inadequate oxygen supply rapidly in high concentrations which is more effective than the delivery of oxygen by haemoglobin.

In patients with severe anemia who experience oxygen debt, intermitten HBOT can fill the 'debt' until the haemoglobin levels back to their normal function. Although not shown specifically in COVID-19 patients, oxygen debt mainly occurs due to a prolonged period of hypoxemia that can be treated by HBOT. The ability to reverse oxygen debt states an important effect of HBOT in the management of patients with tissue oxygen insufficiency. In the other hand, tissue hypoxia is an uncommon cause of increased blood lactate levels as a potential marker of oxygen debt in sepsis, especially after sufficient initial resuscitation[95][96]. Increased oxygen supply ultimately maintains cellular metabolism and organ function. HBOT is also reported to improve mitochondrial function as well as repair balance between glycolysis and mitochondrial respiration, making it possible to counteract the effects of viral infection on tissue caloristasis cell[97] in COVID-19 patients. Further research should be carried out to analyze whether payment of oxygen debt is a key mechanism for HBOT on COVID-19.

Increased systemic oxygen levels using HBOT are often confused with the term metabolic effect of respiration with transient hyperoxemia which disappears as soon as the patient leaves the chamber. However, immediate and continued tropic effects of HBOT have been documented in both single and repetitive HBOT 
administration ever since 1960s[79]. One of the mechanisms recently described is epigenetic modulation as a direct effect of hydrostatic pressure and hyperoxia on more than $40 \%$ of protein coding genes in the human genome. The largest upregulated genes clusters are growth, repair, cell signaling and anti-inflammatory genes and the downregulated gene clusters are proinflammatory genes and genes that control programmed cell death.

Two important transcription factors induced by HBOT at a pressure of 2,4 ATA are Nrf-2 and Heat shock transcription factor 1 (HSF-1). Nrf-2 stimulates hundreds of cell defence proteins, most of which participate in the oxidative stress response. HSF-1 induces cells to produce additional defence proteins that are antiinflammatory[98]. The ability of HBOT to reduce the inflammatory response[84][99] has been demonstrated in several animal model studies using TNF- $\alpha$ and IL-6 inflammatory indicators in femoral head avascular necrosis[100], IL-1 $\beta$ and NLRP3 in a traumatic brain injury model[101], pancreatitis[102][103][104], sepsis[99] and colitis ulcerative[105] .

Although HBOT is a relatively safe therapy and can be used in conjunction with mechanical ventilation[106], it still has some side effects to take into consideration especially in COVID -19 patients. Oxygen toxicity is the most dangerous side effects for COVID-19 patients since they have received high concentrations of normobaric oxygen therapy which can also trigger oxygen toxicity even before entering the hyperbaric chamber. Moreover, the symptoms of oxygen toxicity are non specific (cough, chest thightness) that already exist in COVID-19 patient. However, for some patients that have been treated with HBOT, they do not show any acute worsening due to therapy although there are no specific measurements that can distinguish lung damage due to the disease itself or oxygen toxicity caused by HBOT in patients. The UHMS Committee thinks that if there is an adverse effect due to oxygen toxicity then it will worsen the outcome in the hyperbaric group, and this is consistent with observation by $\mathrm{Dr}$ Thibodeaux in UHMS webinar (July $20^{\text {th }}$ 2020)[18]. There are currently 8 local and international RCT studies on the use of HBOT for COVID - 19 patient which can be accessed through clinicaltrial.gov.

\section{COUNTRIES USING HBOT AS} ADJUVANT THERAPY FOR COVID 19 PATIENT

\section{China}

Two articles that have been published in China show that HBOT has the potential to be used in COVID - 19 patients with pneumonia.

The first is a case report from a patient with very severe symptoms who experienced respiratory failure (not intubated) and the symptoms improved after 8 times HBOT at $200 \mathrm{kPa}$ for 95 minutes (Zong $\mathrm{X}$ et al in Harch PG, 2020)[107].

Second is a patient on ventilator with more severe symptoms of disease with ARDS which is saved by using HBOT. Four other COVID-19 patients had severe symptoms with ground glass opacities that could no longer be treated using standard oxygen masks treated with 3-5 times HBOT and returned home. Then by the same researchers they also performed therapy on 20 other patients with milder symptoms and gave the same outcome. Five patients who were not intubated had used oxygen for several days or even weeks with their preHBOT oxygen saturation $70 \%$ (using oxygen mask). With daily HBOT administration, patient experienced a persistent increase in oxygen saturation an improvement in symptoms the day after. With only 3- 8 times HBOT the patient can go through the hypoxic crisis phase of the disease and be able to be discharged from the hospital. Researchers suggest that giving HBOT at the initial process of the disease can prevent worsening condition which can lead to significant morbidity and mortality of COVID-19 infection (Zong X et al in Harch PG, 2020)[107]. 
During therapy in 35 COVID-19 patients, none of their staff were infected because infection control procedures were carried out in the hyperbaric department and in the chamber itself to avoid cross contamination between health workers and other patients. This is applaudable considering that transmission to health workers reaches a significant number in hospitals treating COVID-19 patientss both in China and in The USA[107].

\section{USA}

Thibodeaux[108] reported giving HBOT in 5 patients from April $13^{\text {th }}$ to $20^{\text {th }}$ with symptoms of tachypnoea and low oxygen saturation. The patient were given a high $\mathrm{FiO}$ and the administration of HBOT is to avoid the use of mechanical ventilation. The therapy given was 2 ATA for 90 minutes nonstop in a monoplace chamber. Therapy is given $1-6$ times. The result obtained were all patients were not on a ventilator and there was a rapid improvement in tachypnoea and an increase in oxygen saturation. Within less than one week from the last therapy, 3 patients were discharged and 2 were still being treated in a stable condition.

Gorenstein made a report on HBOT therapy for 20 COVID 19 patients using matching control s. Therapy is given at a pressure of 2 ATA for 90 minutes every day for a maximum of 5 days. The result obtained were $10 \%$ (2 patients) still need intubation and died while the others were not hospitalized. Meanwhile, in the control group, it was found that $30 \%$ required intubation, $22 \%$ died and $5 \%$ remain hospitalized. They concluded that HBOT is safe and effective as an adjuvant therapy in COVID-19 patients[109].

Until now COVID-19 has not been included in the list of indications for the use of HBOT. Therefor European Committee for Hyperbaric Medicine takes a precautionary position where the use of HBOT must be considered per case and adjusted to the judgment of attending physician[110], [111].

\section{CONCLUSION}

Although mechanical ventilator as used in COVID 19 patients with severe hypoxia in the ICU and HBOT can increase arterial oxygen levels, HBOT has advantages over ventilator. Other than anti-inflammatory properties, high pressure ambient and the use of $100 \%$ oxygen guarantee increased oxygen partial pressure in circulation and tissue despite decreased aerated pulmonary in COVID -19 patient with ARDS. With oxygen debt being 'payed' by HBOT it will reverse tissue hypoxia and then the probability of a patient using mechanical ventilation also decreased. However, until now there is still no recommendation of HBOT in COVID-patient until the trial is completed.

\section{REFERENCES}

[1] H. Harapan et al., "Coronavirus disease 2019 (COVID-19): A literature review," J. Infect. Public Health, vol. 13, no. 5, pp. 667-673, 2020, doi: 10.1016/j.jiph.2020.03.019.

[2] Z. Wu and J. M. McGoogan, "Characteristics of and Important Lessons from the Coronavirus Disease 2019 (COVID-19) Outbreak in China: Summary of a Report of 72314 Cases from the Chinese Center for Disease Control and Prevention," JAMA - J. Am. Med. Assoc., vol. 323, no. 13, pp. 1239-1242, 2020, doi: 10.1001/jama.2020.2648.

[3] S. R. Weiss and S. Navas-Martin, "Coronavirus Pathogenesis and the Emerging Pathogen Severe Acute Respiratory Syndrome Coronavirus," Microbiol. Mol. Biol. Rev., vol. 69, no. 4, pp. 635-664, 2005, doi: 10.1128/mmbr.69.4.635-664.2005.

[4] Q. Ye, B. Wang, and J. Mao, "The pathogenesis and treatment of the 'Cytokine Storm"' in COVID-19,"' Journal of Infection. 2020, doi:

10.1016/j.jinf.2020.03.037.

[5] D. E. Fry, L. Pearlstein, R. L. Fulton, and H. C. Polk, "Multiple System Organ Failure: The Role of Uncontrolled Infection," Arch. Surg., vol. 115, no. 2, pp. 136-140, 1980, doi: 10.1001/archsurg.1980.01380020006003.

[6] J. Heugel, E. T. Martin, J. Kuypers, and J. A. Englund, "Coronavirus-associated pneumonia in previously healthy children," 
Pediatr. Infect. Dis. J., 2007, doi: 10.1097/INF.0b013e318054e31b.

[7] S. Perlman and J. Netland, "Coronaviruses post-SARS: Update on replication and pathogenesis," Nat. Rev. Microbiol., vol. 7, no. 6 , pp. 439-450, 2009, doi:

10.1038/nrmicro2147.

[8] S. Perlman and A. A. Dandekar, "Immunopathogenesis of coronavirus infections: Implications for SARS," Nat. Rev. Immunol., vol. 5, no. 12, pp. 917-927, 2005, doi: 10.1038/nri1732.

[9] B. Sainz, E. C. Mossel, C. J. Peters, and R. F. Garry, "Interferon-beta and interferongamma synergistically inhibit the replication of severe acute respiratory syndrome-associated coronavirus (SARSCoV)," Virology, vol. 329, no. 1, pp. 1117, 2004, doi: 10.1016/j.virol.2004.08.011.

[10] L. Velazquez-Salinas, A. VerdugoRodriguez, L. L. Rodriguez, and M. V. Borca, "The role of interleukin 6 during viral infections," Front. Microbiol., vol. 10, no. MAY, pp. 6-11, 2019, doi: 10.3389/fmicb.2019.01057.

[11] M. Zheng et al., "Functional exhaustion of antiviral lymphocytes in COVID-19 patients," Cell. Mol. Immunol., vol. 17, no. 5, pp. 533-535, 2020, doi: 10.1038/s41423020-0402-2.

[12] W. Zhang et al., "Molecular and serological investigation of 2019-nCoV infected patients: implication of multiple shedding routes," Emerg. Microbes Infect., 2020, doi:

10.1080/22221751.2020.1729071.

[13] C. S. Deutschman and K. J. Tracey, "Sepsis: Current dogma and new perspectives," Immunity, vol. 40, no. 4, pp. 463-475, 2014, doi:

10.1016/j.immuni.2014.04.001.

[14] M. Singer et al., "The third international consensus definitions for sepsis and septic shock (sepsis-3)," JAMA - J. Am. Med. Assoc., vol. 315, no. 8, pp. 801-810, 2016, doi: 10.1001/jama.2016.0287.

[15] C. M. Coopersmith and C. S. Deutschman, "The new sepsis definitions: Implications for the basic and translational research communities," Shock, vol. 47, no. 3, pp. 264-268, 2017, doi: 10.1097/SHK.0000000000000763.

[16] K. J. Huang et al., "An interferon- $\gamma$-related cytokine storm in SARS patients," J. Med. Virol., vol. 75, no. 2, pp. 185-194, 2005, doi: 10.1002/jmv.20255.

[17] V. M. Ranieri et al., "Acute respiratory distress syndrome: The Berlin definition," JAMA - J. Am. Med. Assoc., vol. 307, no. 23, pp. 2526-2533, 2012, doi: 10.1001/jama.2012.5669.

[18] J. J. Feldmeier et al., "Rationale, Study Design Considerations, and Protocol Recommendations for Treating Covid-19 Patients With Hyperbaric Oxygen," pp. 135, 2020.

[19] R. Pardal and P. Ortega-s, "Cellular Mechanisms of Oxygen Sensing," Annu rev physiol, vol. 63, pp. 259-87, 2001.

[20] D. G. Hardie, "Minireview: The AMPActivated Protein Kinase Cascade: The Key Sensor of Cellular Energy Status," Endocrinology, vol. 144, no. 12, pp. 51795183, 2003, doi: 10.1210/en.2003-0982.

[21] F. H. Epstein, H. L. Manning, and R. M. Schwartzstein, "Pathophysiology of dyspnea," New England Journal of Medicine. 1995, doi:

10.1056/NEJM199512073332307.

[22] K. Vaporidi, E. Akoumianaki, I. Telias, E. C. Goligher, L. Brochard, and D. Georgopoulos, "Respiratory drive in critically Ill patients pathophysiology and clinical implications," Am. J. Respir. Crit. Care Med., vol. 201, no. 1, pp. 20-32, 2020, doi: 10.1164/rccm.201903-0596SO.

[23] S. Dhont, E. Derom, E. Van Braeckel, P. Depuydt, and B. N. Lambrecht, "The pathophysiology of 'happy' hypoxemia in COVID-19," Respir. Res., vol. 21, no. 1, p. 198, 2020, doi: 10.1186/s12931-02001462-5.

[24] P. A. Easton, L. J. Slykerman, and N. R. Anthonisen, "Ventilatory response to sustained hypoxia in normal adults," $J$. Appl. Physiol., vol. 61, no. 3, pp. 906-911, 1986, doi: 10.1152/jappl.1986.61.3.906.

[25] P. A. Kvale, W. A. Conway, and E. O. Coates, "Continuous or nocturnal oxygen therapy in hypoxemic chronic obstructive lung disease. A clinical trial," Ann. Intern. Med., 1980, doi: 10.7326/0003-4819-93-3391.

[26] S. C. Gandevia et al., "Respiratory sensations, cardiovascular control, kinaesthesia and transcranial stimulation during paralysis in humans.," J. Physiol., 1993, doi: 10.1113/jphysiol.1993.sp019849.

[27] L. Gattinoni et al., "COVID-19 pneumonia: different respiratory treatments for different phenotypes?," Intensive Care Med., vol. 46, no. 6, pp. 1099-1102, 2020, doi: 10.1007/s00134-020-06033-2.

[28] F. R. Sharp et al., "Hypoxic Preconditioning Protects against Ischemic Brain Injury," NeuroRx, 2004, doi: 10.1602/neurorx.1.1.26.

[29] K. Biagas, "Hypoxic-ischemic brain injury: Advancements in the understanding of 
mechanisms and potential avenues for therapy," Current Opinion in Pediatrics, vol. 11, no. 3. pp. 223-228, 1999, doi: 10.1097/00008480-199906000-00009.

[30] A. Zauner, W. P. Daugherty, M. R. Bullock, D. S. Warner, W. R. Selman, and R. J. Dempsey, "Brain oxygenation and energy metabolism: Part I - Biological function and pathophysiology," Neurosurgery. 2002, doi: 10.1097/00006123-200208000-00003.

[31] M. C. Brahimi-Horn and J. Pouysségur, "Oxygen, a source of life and stress," FEBS Lett., vol. 581, no. 19, pp. 3582-3591, 2007, doi: 10.1016/j.febslet.2007.06.018.

[32] E. C. Toescu, "Hypoxia sensing and pathways of cytosolic $\mathrm{Ca} 2+$ increases," Cell Calcium, vol. 36, no. 3-4, pp. 187 199, 2004, doi: 10.1016/j.ceca.2004.02.019.

[33] K. A. Lee, R. A. Roth, and J. J. LaPres, "Hypoxia, drug therapy and toxicity," Pharmacol. Ther., vol. 113, no. 2, pp. 229 246, 2007, doi: 10.1016/j.pharmthera.2006.08.001.

[34] I. R. LEUSEN, "Chemosensitivity of the respiratory center; influence of $\mathrm{CO} 2$ in the cerebral ventricles on respiration," Am. J. Physiol., 1954, doi: 10.1152/ajplegacy.1953.176.1.39.

[35] P. G. Guyenet and D. A. Bayliss, "Neural Control of Breathing and $\mathrm{CO} 2$ Homeostasis," Neuron. 2015, doi: 10.1016/j.neuron.2015.08.001.

[36] L. C. Mchenry, H. C. Slocum, H. E. Bivens, H. A. Mayes, and G. J. Hayes, "Hyperventilation in Awake and Anesthetized Man: Effects on Cerebral Blood Flow and Cerebral Metabolism," Arch. Neurol., 1965, doi: 10.1001/archneur.1965.00460270046006.

[37] L. Meng and A. W. Gelb, "Regulation of cerebral autoregulation by carbon dioxide," Anesthesiology, vol. 122, no. 1, pp. 196205, 2015, doi: 10.1097/ALN.0000000000000506.

[38] W. Guan et al., "Clinical characteristics of coronavirus disease 2019 in China," $N$. Engl. J. Med., vol. 382, no. 18, pp. 17081720, 2020, doi: 10.1056/NEJMoa2002032.

[39] G. Allali, C. Marti, O. Grosgurin, C. Morélot-Panzini, T. Similowski, and D. Adler, "Dyspnea: The vanished warning symptom of COVID-19 pneumonia," $J$. Med. Virol., pp. 1-2, 2020, doi: $10.1002 / j m v .26172$.

[40] J. Couzin-Frankel, "The mystery of the pandemic's 'happy hypoxia," Science (80. )., vol. 368, no. 6490, pp. 455-456, 2020.
[41] R. G. Wilkerson, J. D. Adler, N. G. Shah, and R. Brown, "Silent hypoxia: A harbinger of clinical deterioration in patients with COVID-19," Am. J. Emerg. Med., 2020, doi: 10.1016/j.ajem.2020.05.044.

[42] J. Xie, Z. Tong, X. Guan, B. Du, H. Qiu, and A. S. Slutsky, "Critical care crisis and some recommendations during the COVID19 epidemic in China," Intensive Care Med., vol. 46, no. 5, pp. 837-840, 2020, doi: 10.1007/s00134-020-05979-7.

[43] M. J. Tobin, F. Laghi, and A. Jubran, "Why COVID-19 Silent Hypoxemia Is Baffling to Physicians," Am. J. Respir. Crit. Care Med., vol. 202, no. 3, pp. 356-360, 2020, doi: $10.1164 / \mathrm{rccm} .202006-2157 \mathrm{CP}$.

[44] A. De Maio and L. E. Hightower, "COVID-19, acute respiratory distress syndrome (ARDS), and hyperbaric oxygen therapy (HBOT): what is the link?," Cell Stress Chaperones, 2020, doi: 10.1007/s12192-020-01121-0.

[45] M. L. Fung, "Expressions of angiotensin and cytokine receptors in the paracrine signaling of the carotid body in hypoxia and sleep apnea," Respir. Physiol. Neurobiol., vol. 209, pp. 6-12, 2015, doi: 10.1016/j.resp.2014.09.014

[46] A. R. Sedaghat, I. Gengler, and M. M. Speth, "Olfactory Dysfunction: A Highly Prevalent Symptom of COVID-19 With Public Health Significance," Otolaryngol. Head Neck Surg. (United States), vol. 163, no. 1, pp. 12-15, 2020, doi: 10.1177/0194599820926464.

[47] J. Netland, D. K. Meyerholz, S. Moore, M. Cassell, and S. Perlman, "Severe Acute Respiratory Syndrome Coronavirus Infection Causes Neuronal Death in the Absence of Encephalitis in Mice Transgenic for Human ACE2," J. Virol., vol. 82 , no. 15 , pp. $7264-7275$, 2008, doi: 10.1128/jvi.00737-08.

[48] G. E. D'Alonzo and D. R. Dantzker, "Respiratory failure, mechanisms of abnormal gas exchange, and oxygen delivery," Med. Clin. North Am., vol. 67, no. 3, pp. 557-571, 1983, doi: 10.1016/S0025-7125(16)31189-0.

[49] S. L. Archer, W. W. Sharp, and E. K. Weir, "Differentiating COVID-19 Pneumonia from Acute Respiratory Distress Syndrome and High Altitude Pulmonary Edema: Therapeutic Implications," Circulation, pp. 101-104, 2020, doi: 10.1161/CIRCULATIONAHA.120.047915

[50] M. Lang et al., "Hypoxaemia related to COVID-19: vascular and perfusion 
abnormalities on dual-energy CT," The

Lancet Infectious Diseases. 2020, doi: 10.1016/S1473-3099(20)30367-4.

[51] W. Ottestad and S. Søvik, "COVID-19 patients with respiratory failure: what can we learn from aviation medicine?," British Journal of Anaesthesia. 2020, doi: 10.1016/j.bja.2020.04.012.

[52] C. Nagaraj et al., "Hypoxic vascular response and ventilation/perfusion matching in end-stage COPD may depend on p22phox," Eur. Respir. J., vol. 50, no. 1, 2017, doi: 10.1183/13993003.01651-2016.

[53] M. Vaduganathan, O. Vardeny, T. Michel, J. J. V. McMurray, M. A. Pfeffer, and S. D. Solomon, "Renin-angiotensin-aldosterone system inhibitors in patients with covid19," N. Engl. J. Med., 2020, doi: 10.1056/NEJMsr2005760.

[54] S. Wang et al., "Endocytosis of the receptor-binding domain of SARS-CoV spike protein together with virus receptor ACE2," Virus Res., vol. 136, no. 1-2, pp. 8-15, 2008, doi: 10.1016/j.virusres.2008.03.004.

[55] M. Z. Tay, C. M. Poh, L. Rénia, P. A. MacAry, and L. F. P. Ng, "The trinity of COVID-19: immunity, inflammation and intervention," Nat. Rev. Immunol., vol. 20, no. 6, pp. 363-374, 2020, doi: 10.1038/s41577-020-0311-8.

[56] Y. Liu et al., "Clinical and biochemical indexes from 2019-nCoV infected patients linked," Sci. China Life Sci., vol. 63, no. 3, pp. 364-374, 2020, doi: 10.1007/s11427020-1643-8.

[57] H. P. Jia et al., “ACE2 Receptor Expression and Severe Acute Respiratory Syndrome Coronavirus Infection Depend on Differentiation of Human Airway Epithelia," J. Virol., vol. 79, no. 23, pp. 14614-14621, 2005, doi: 10.1128/jvi.79.23.14614-14621.2005.

[58] B. Bikdeli et al., "COVID-19 and Thrombotic or Thromboembolic Disease: Implications for Prevention, Antithrombotic Therapy, and Follow-Up: JACC State-of-the-Art Review," J. Am. Coll. Cardiol., vol. 75, no. 23, pp. 29502973, 2020, doi:

10.1016/j.jacc.2020.04.031.

[59] C. Guérin and M. A. Matthay, "Acute cor pulmonale and the acute respiratory distress syndrome," Intensive Care Med., vol. 42, no. 5, pp. 934-936, 2016, doi: 10.1007/s00134-015-4197-z.

[60] C. M. Campbell and R. Kahwash, "Will Complement Inhibition Be the New Target in Treating COVID-19-Related Systemic Thrombosis?," Circulation, pp. 1739-1741,
2020, doi: 10.1161/CIRCULATIONAHA.120.047419

[61] L. Zhang et al., "D-dimer levels on admission to predict in-hospital mortality in patients with Covid-19," J. Thromb. Haemost., vol. 18, no. 6, pp. 1324-1329, 2020, doi: 10.1111/jth.14859.

[62] J. J. Marini and L. Gattinoni, "Management of COVID-19 Respiratory Distress," JAMA - Journal of the American Medical Association. 2020, doi: 10.1001/jama.2020.6825.

[63] J. Wang et al., "Tissue plasminogen activator (tPA) treatment for COVID-19 associated acute respiratory distress syndrome (ARDS): A case series," $J$. Thromb. Haemost., vol. 18, no. 7, pp. 1752-1755, 2020, doi: 10.1111/jth.14828.

[64] J. Á. Monsalve-Naharro, E. DomingoChiva, S. G. Castillo, P. Cuesta-Montero, and J. M. Jiménez-Vizuete, "Inhaled nitric oxide in adult patients with acute respiratory distress syndrome," Farm. Hosp., vol. 41, no. 2, pp. 292-312, 2017, doi: $10.7399 /$ fh.2017.41.2.10533.

[65] W. Luo et al., "Clinical pathology of critical patient with novel coronavirus pneumonia (COVID-19)," Preprints, 2020, doi: 10.13140/RG.2.2.22934.29762.

[66] S. Tian, W. Hu, L. Niu, H. Liu, H. Xu, and S. Y. Xiao, "Pulmonary Pathology of Early-Phase 2019 Novel Coronavirus (COVID-19) Pneumonia in Two Patients With Lung Cancer," J. Thorac. Oncol., 2020, doi: 10.1016/j.jtho.2020.02.010.

[67] R. J. Mason, "Pathogenesis of COVID-19 from a cell biology perspective," Eur. Respir. J., vol. 55, no. 4, 2020, doi: 10.1183/13993003.00607-2020.

[68] S. R. Hopkins, "Exercise induced arterial hypoxemia: The role of ventilationperfusion inequality and pulmonary diffusion limitation," Adv. Exp. Med. Biol., vol. 588, pp. 17-30, 2006, doi: 10.1007/978-0-387-34817-9_3.

[69] D. R. Ziehr et al., "Respiratory pathophysiology of mechanically ventilated patients with COVID-19: A cohort study," Am. J. Respir. Crit. Care Med., vol. 201, no. 12, pp. 1560-1564, 2020, doi: 10.1164/rccm.202004-1163LE.

[70] C. Hamilton, B. Steinlechner, E. Gruber, P. Simon, and G. Wollenek, "The oxygen dissociation curve: Quantifying the shift," Perfusion, vol. 19, no. 3, pp. 141-144, 2004, doi: 10.1191/0267659104pf734oa.

[71] S. Woyke, S. Rauch, M. Ströhle, and H. Gatterer, "Modulation of Hb-O2 affinity to improve hypoxemia in COVID-19 
patients," Clin. Nutr., 2020, doi:

10.1016/j.clnu.2020.04.036.

[72] J. Xie et al., "Association Between

Hypoxemia and Mortality in Patients With

COVID-19," Mayo Clin. Proc., vol. 95, no.

6, pp. 1138-1147, 2020, doi:

10.1016/j.mayocp.2020.04.006.

[73] H. K. Siddiqi and M. R. Mehra, "COVID-

19 illness in native and immunosuppressed states: A clinical-therapeutic staging proposal," J. Hear. Lung Transplant., vol. 39, no. 5, pp. 405-407, 2020, doi:

10.1016/j.healun.2020.03.012.

[74] M. Komorowski and S. K. Aberegg, "Using applied lung physiology to understand COVID-19 patterns," British

Journal of Anaesthesia. 2020, doi: 10.1016/j.bja.2020.05.019.

[75] L. Gattinoni, S. Coppola, M. Cressoni, M. Busana, S. Rossi, and D. Chiumello, "COVID-19 does not lead to a 'typical' acute respiratory distress syndrome," Am. J. Respir. Crit. Care Med., vol. 201, no. 10, pp. 1299-1300, 2020, doi:

10.1164/rccm.202003-0817LE.

[76] L. Gattinoni, D. Chiumello, and S. Rossi, "COVID-19 pneumonia: ARDS or not?," Crit. Care, vol. 24, no. 1, pp. 1-3, 2020, doi: 10.1186/s13054-020-02880-z.

[77] D. Wichmann et al., "Autopsy Findings and Venous Thromboembolism in Patients With COVID-19," Ann. Intern. Med., vol. 25, no. 4, 2020, doi: 10.7326/m20-2003.

[78] O. Sanchez et al., "Pathophysiology of dyspnoea in acute pulmonary embolism: A cross-sectional evaluation," Respirology, vol. 22 , no. 4, pp. 771-777, 2017, doi: 10.1111/resp.12961.

[79] K. K. Jain and K. K. Jain, The History of Hyperbaric Medicine. 2017.

[80] D. Rixen and J. H. Siegel, "Bench-tobedside review: Oxygen debt and its metabolic correlates as quantifiers of the severity of hemorrhagic and posttraumatic shock," Crit. Care, vol. 9, no. 5, pp. 441453, 2005, doi: 10.1186/cc3526.

[81] D. M. Cauvi et al., "Period of irreversible therapeutic intervention during sepsis correlates with phase of innate immune dysfunction," J. Biol. Chem., vol. 287, no. 24, pp. 19804-19815, 2012, doi: 10.1074/jbc.M112.359562.

[82] D. M. Cauvi, D. Hawisher, P. R. DoresSilva, R. E. Lizardo, and A. De Maio, "Macrophage reprogramming by negatively charged membrane phospholipids controls infection," FASEB J., vol. 33, no. 2, pp. 2995-3009, 2019, doi: 10.1096/fj.201801579R.

[83] M. A. Puskarich, M. R. Marchick, J. A.
Kline, M. T. Steuerwald, and A. E. Jones, "One year mortality of patients treated with an emergency department based early goal directed therapy protocol for severe sepsis and septic shock: A before and after study," Crit. Care, vol. 13, no. 5, pp. 1-7, 2009, doi: $10.1186 /$ cc8138.

[84] J. L. Halbach et al., "Early hyperbaric oxygen therapy improves survival in a model of severe sepsis," Am. J. Physiol. Regul. Integr. Comp. Physiol., vol. 317, no. 1, pp. R160-R168, 2019, doi: 10.1152/ajpregu.00083.2019.

[85] A. L. Gill and C. N. A. Bell, "Hyperbaric oxygen: Its uses, mechanisms of action and outcomes," QJM - Mon. J. Assoc.

Physicians, vol. 97, no. 7, pp. 385-395, 2004, doi: 10.1093/qjmed/hch074.

[86] R. M. Stoekenbroek, T. B. Santema, D. A. Legemate, D. T. Ubbink, A. Van Den Brink, and M. J. W. Koelemay, "Hyperbaric oxygen for the treatment of diabetic foot ulcers: A systematic review," Eur. J. Vasc. Endovasc. Surg., vol. 47, no. 6, pp. 647-655, 2014, doi: 10.1016/j.ejvs.2014.03.005.

[87] M. Edremitlioğlu et al., "The effect of hyperbaric oxygen treatment on the renal functions in septic rats: Relation to oxidative damage," Surg. Today, vol. 35, no. 8, pp. 653-661, 2005, doi: 10.1007/s00595-004-3000-5.

[88] L. E. Harrison, C. Giardina, L. E. Hightower, C. Anderson, and G. A. Perdrizet, "Might hyperbaric oxygen therapy (HBOT) reduce renal injury in diabetic people with diabetes mellitus? From preclinical models to human metabolomics," Cell Stress Chaperones, vol. 23, no. 6, pp. 1143-1152, 2018, doi: 10.1007/s12192-018-0944-8.

[89] J. P. Kirby, "Hyperbaric Oxygen Therapy and Radiation-Induced Injuries.," Mo. Med., vol. 116, no. 3, pp. 198-200, 2019.

[90] J. A. Buras and W. R. Reenstra, "Endothelial-neutrophil interactions during ischemia and reperfusion injury: Basic mechanisms of hyperbaric oxygen," Neurol. Res., vol. 29, no. 2, pp. 127-131, 2007, doi: 10.1179/016164107X174147.

[91] J. Kirby, "Hyperbaric Oxygen Therapy and Negative Pressure as Advanced Wound Management.," Mo. Med., vol. 116, no. 3, pp. 192-194, 2019.

[92] A. M. Fuller, C. Giardina, L. E. Hightower, G. A. Perdrizet, and C. A. Tierney, "Hyperbaric oxygen preconditioning protects skin from UV-A damage," Cell Stress Chaperones, 2013, doi: 10.1007/s12192-012-0362-2. 
[93] G. G. Rogatsky, E. G. Shifrin, and A. Mayevsky, "Acute respiratory distress syndrome in patients after blunt thoracic trauma: The influence of hyperbaric oxygen therapy," Adv. Exp. Med. Biol., vol. 540, pp. 77-85, 2003, doi: 10.1007/978-14757-6125-2_12.

[94] L. M. Sellers, "The fallibility of the forrestian principle," Laryngoscope, vol. 74, no. 5. pp. 613-633, 1964, doi: 10.1288/00005537-196405000-00001.

[95] L. Gattinoni et al., "Understanding lactatemia in human sepsis potential impact for early management," Am. J. Respir. Crit. Care Med., vol. 200, no. 5, pp. 582-589, 2019, doi: 10.1164/rccm.201812-2342OC.

[96] G. Hernandez, R. Bellomo, and J. Bakker, "The ten pitfalls of lactate clearance in sepsis," Intensive Care Med., vol. 45, no. 1, pp. 82-85, 2019, doi: 10.1007/s00134-0185213-x.

[97] D. Tezgin, C. Giardina, G. A. Perdrizet, and L. E. Hightower, "The effect of hyperbaric oxygen on mitochondrial and glycolytic energy metabolism: the caloristasis concept," Cell Stress Chaperones, vol. 25, no. 4, pp. 667-677, 2020, doi: 10.1007/s12192-020-01100-5.

[98] C. A. Godman, K. P. Chheda, L. E. Hightower, G. Perdrizet, D. G. Shin, and C. Giardina, "Hyperbaric oxygen induces a cytoprotective and angiogenic response in human microvascular endothelial cells," Cell Stress Chaperones, vol. 15, no. 4, pp. 431-442, 2010, doi: 10.1007/s12192-0090159-0.

[99] J. A. Buras, D. Holt, D. Orlow, B. Belikoff, S. Pavlides, and W. R. Reenstra, "Hyperbaric oxygen protects from sepsis mortality via an interleukin-10-dependent mechanism," Crit. Care Med., vol. 34, no. 10, pp. 2624-2629, 2006, doi: 10.1097/01.CCM.0000239438.22758.E0.

[100] G. Bosco et al., "Hyperbaric oxygen therapy ameliorates osteonecrosis in patients by modulating inflammation and oxidative stress," J. Enzyme Inhib. Med. Chem., 2018, doi: 10.1080/14756366.2018.1485149.

[101] H. Qian, Q. Li, and W. Shi, "Hyperbaric oxygen alleviates the activation of NLRP3 -inflammasomes in traumatic brain injury," Mol. Med. Rep., vol. 16, no. 4, pp. 3922-3928, 2017, doi: 10.3892/mmr.2017.7079.

[102] C. Christophi, I. Millar, M. Nikfarjam, V. Muralidharan, and C. Malcontenti-Wilson, "Hyperbaric oxygen therapy for severe acute pancreatitis," J. Gastroenterol.
Hepatol., vol. 22, no. 11, pp. 2042-2046, 2007, doi: 10.1111/j.1440-

1746.2006.03380.x.

[103] M. Nikfarjam, C. M. Cuthbertson, C. Malcontenti-Wilson, V. Muralidharan, I. Millar, and C. Christophi, "Hyperbaric oxygen therapy reduces severity and improves survival in severe acute pancreatitis," J. Gastrointest. Surg., vol. 11, no. 8, pp. 1008-1015, 2007, doi: 10.1007/s11605-007-0175-2.

[104] X. Bai et al., "Down-regulation of hypoxiainducible factor- $1 \alpha$ by hyperbaric oxygen attenuates the severity of acute pancreatitis in rats," Pancreas, vol. 38, no. 5, pp. 515522, 2009, doi: 10.1097/MPA.0b013e31819cac24.

[105] P. S. Dulai and V. Jairath, "Acute severe ulcerative colitis: latest evidence and therapeutic implications," Ther. Adv. Chronic Dis., vol. 9, no. 2, pp. 65-72, 2018, doi: 10.1177/2040622317742095.

[106] J. Bessereau et al., "Safety of hyperbaric oxygen therapy in mechanically ventilated patients," Int. Marit. Health, vol. 68, no. 1, pp. 46-51, 2017, doi: 10.5603/IMH.2017.0008.

[107] P. Harch, "Hyperbaric oxygen treatment of novel coronavirus (COVID-19) respiratory failure," Med. Gas Res., vol. 10, no. 2, pp. 61-62, 2020, doi: 10.4103/20459912.282177

[108] K. Thibodeaux, M. Speyrer, A. Raza, R. Yaakov, and T. E. Serena, "Hyperbaric oxygen therapy in preventing mechanical ventilation in COVID-19 patients: a retrospective case series," J. Wound Care, vol. 29, no. Sup5a, pp. 5-8, 2020, doi: 10.12968/jowc.2020.29.Sup5a.S4.

[109] S. A. Gorenstein et al., "Hyperbaric oxygen therapy for covid-19 patients with respiratory distress: treated cases versus propensity-matched controls," uhms, vol. 47, no. 3, pp. 405-413, 2020.

[110] T. J. Francis et al., "ECHM position on Hyperbaric Oxygen Therapy (HBOT) in multiple Hyperbaric chambers during coronavirus disease (COVID-19) Outbreak," no. 16th march 2020. 2020.

[111] S. R. T. Matteo Paganini, Gerardo Bosco, Filippo A. G. Perozzo, Eva Kohlscheen, Regina Sonda, Franco Bassetto, Giacomo Garetto, Enrico M. Camporesi, "The Role of Hyperbaric Oxygen Treatment for COVID-19: A Review," in Adv Exp Med Biol - Clinical and Experimental Biomedicine, 2020, pp. 57-66. 